\title{
Imagen de la Física universitaria: el punto de vista del profesor y del alumno
}

\author{
F. J. Perales'; J. A. García ${ }^{2}$; R. Huertas ${ }^{2}$; \\ L. Gómez-Robledo ${ }^{2}$ \\ ${ }^{1}$ Departamento de Didáctica de las Ciencias Experimentales \\ ${ }^{2}$ Departamento de Óptica \\ Universidad de Granada
}

Resumen: Este trabajo pretende indagar en algunos factores cognitivos y actitudinales que inciden en la elección y adaptación de los alumnos que ingresan en carreras científicas. Para ello se parte de una revisión teórica sobre las actitudes hacia la ciencia y la orientación vocacional. Posteriormente se describen los resultados de sendas encuestas realizadas a alumnos de Ingeniería Química y de Óptica, así como a algunos profesores, una de ellas diferida en un periodo de cinco años para alumnos de esta última titulación y la otra aplicada tras la visualización de algunas experiencias de laboratorio. Se discuten los resultados de los distintos item, tanto para cada muestra como comparativamente, y se reflexiona sobre posibles alternativas para mejorar la elección de carreras científicas.

Palabras clave: Actitudes, orientación vocacional, física, óptica, ingeniería.

Abstract: This work aims to research cognitive and attitudinal factors that influence the choice and adaptation of students entering scientific careers. For it a theoretical review on attitudes towards science and vocational guidance is showed. Below the results of different samples of teachers and students of Chemical Engineering and Optics are exposed, one of them deferred over a period of five years to students of this last degree, and the other after viewing some laboratory experiments. The results of the different item, for each sample as both comparatively, are discussed and then a reflection on possible ways to improve scientific career choices is presented.

Key Words: Attitudes, vocational guidance, physics, optics, engineering.

(Fecha de recepción: abril, 2013, y de aceptación: septiembre, 2013)

DOI: 10.7203/DCES.27.2381 


\section{Introducción}

La actual crisis económica que atenaza a los países tradicionalmente en la vanguardia mundial en asuntos de cohesión social como la Educación o la Sanidad, no solo amenaza a estos pilares del "estado del bienestar", está poniendo también en cuestión otros valores de índole moral o profesional, tales como las "vocaciones científicas" que presumiblemente podrían sustentar el desarrollo económico futuro. El consenso en torno a la afirmación de que un estado que apuesta por la Ciencia y la Tecnología está mejor preparado para asumir los retos de la crisis y puede esperar un futuro más halagüeño, parece hacerse trizas cuando los jóvenes universitarios dan la espalda a los estudios científicos.

Voces autorizadas vienen advirtiendo desde hace años acerca de este preocupante hecho y proponiendo algunas iniciativas para revertir esta tendencia, tales como:

- El llamado "Informe Rocard" a nivel europeo (Rocard et al., 2007), que apuesta por una renovación en la forma de enseñar las ciencias, aproximándola a los procesos de investigación científica.

- El reciente "Informe Eurydice" (Comisión Europea, 2011), también de la Unión Europea, afronta un diagnóstico más preciso de la situación y reclama estrategias de mejora más globales: (1) mediante planes de promoción de la educación científica; (2) prolongando las materias de ciencias hasta los últimos cursos de la Educación Secundaria; (3) prestando una atención creciente a la ciencia cotidiana y a las actividades manipulativas; (4) apoyando a los alumnos con un bajo rendimiento en ciencias; (5) renovando los métodos de evaluación tradicionales; y (6) mejorando la formación del profesorado, tanto inicial como permanente.

- En el ámbito español, el "Informe Enciende" (COSCE, 2011) establece un conjunto de cinco propuesta transversales relacionadas con: (1) apoyar la enseñanza de las ciencias desde una perspectiva general (contenidos y su aplicación, metodología y evaluación); (2) un replanteamiento de la formación del profesorado, tanto inicial como permanente, coherente con el punto anterior; (3) el fomento de la cultura científica a nivel familiar y social; (4) introducir la enseñanza de las ciencias en los primeros años de la escolaridad; y (5) coordinar las iniciativas que se alineen con las propuestas anteriores.

Tales iniciativas poseen un carácter "macro" por su carácter institucional $\mathrm{y}$, sin duda, mantienen las suficientes coincidencias como para marcar el rumbo a seguir. No obstante, existen otras variantes del problema desde una perspectiva "micro" y que se pueden relacionar, esencialmente, con las actitudes hacia la ciencia y la propia imagen de ésta entre los estudiantes.

El objetivo de un trabajo como éste es mostrar los resultados de sendas encuestas dirigidas a estudiantes y profesores universitarios que tratan de indagar en algunos de los factores que 
pueden incidir en las actitudes científicas de los primeros.

\section{Marco teórico}

Existe un consenso creciente en torno a la importancia de la vertiente afectiva en el ámbito de la Educación, en general, y de la Didáctica de las Ciencias, en particular (Mellado, Blanco, Borrachero y Cárdenas, 2013; Perales, 2013.). Bajo su paraguas pueden considerarse variables tales como la motivación, el interés, las emociones, el autoconcepto, la autoeficacia, las actitudes, las creencias, los valores, las visiones del mundo (Vázquez, 2013, p. 245).

Una primera diferenciación nos permite hablar de Actitudes hacia la Ciencia y Actitudes Científicas, serán las primeras las que aquí nos ocupen. Vázquez (2013, p. 249) indica sobre ellas que:

"comprenden todos los objetos (acciones, personas, situaciones o ideas) implicados en el aprendizaje de la ciencia (interés por la ciencia, actitudes hacia los científicos (personas) y su trabajo, y actitudes hacia los impactos sociales de la ciencia desde la perspectiva de la responsabilidad social)".

El ámbito de las actitudes hacia la Ciencia posee una larga tradición que entronca con el de las actitudes, en general, estudiado con más profusión por la Psicología Social.

Desde el punto de vista teórico se trata de un tópico complejo que vincula a otros conceptos como el de las creencias, los valores, el autoconcepto... y que ha dado lugar a diferentes mode- lizaciones que tratan de dar cuenta de las relaciones entre ellos. De cualquier forma se suele aceptar que las actitudes poseen tres componentes relacionadas con aspectos cognitivos del objeto actitudinal (saber sobre él), afectivos (predisposición hacia dicho objeto) y comportamentales (acciones concordantes con el mismo).

Señalemos a este respecto, a modo de ejemplo, que la teoría de la expectativavalor trata de explicar los dos factores que contribuyen a la motivación hacia las tareas académicas: en primer lugar, los estudiantes valoran la tarea, ya sea el resultado o el proceso de aprendizaje, y en segundo lugar, su expectativa de éxito. Los estudiantes con motivación de logro (es decir, un aspecto de la valoración de la tarea) aprenden a demostrar que pueden funcionar mejor que otras personas. Las creencias de los estudiantes en su propia eficacia y en lo que ellos atribuyen como éxito y fracaso son los dos grandes temas cuando se habla de las expectativas de éxito. La autoeficacia es por tanto una forma específica de la teoría que explica el autoconcepto, es decir, cómo los individuos se ven a sí mismos. El autoconcepto académico tiene que ver a su vez con cómo los estudiantes ven sus propias capacidades y habilidades en el manejo de las tareas académicas (Bandura, 1977, citado en $\mathrm{Ng}$, Lay, Areepattamannil, Treagust y Chandrasegaran, 2012).

En la figura I se muestra como ejemplo, y de un modo gráfico, las conexiones entre algunos de los conceptos citados para el campo de la formación del profesorado (van Aalderen-Smeets, Walma van der Molen y Asma, 2012). 


\section{FIGURA I. Enseñanza de la ciencia.}

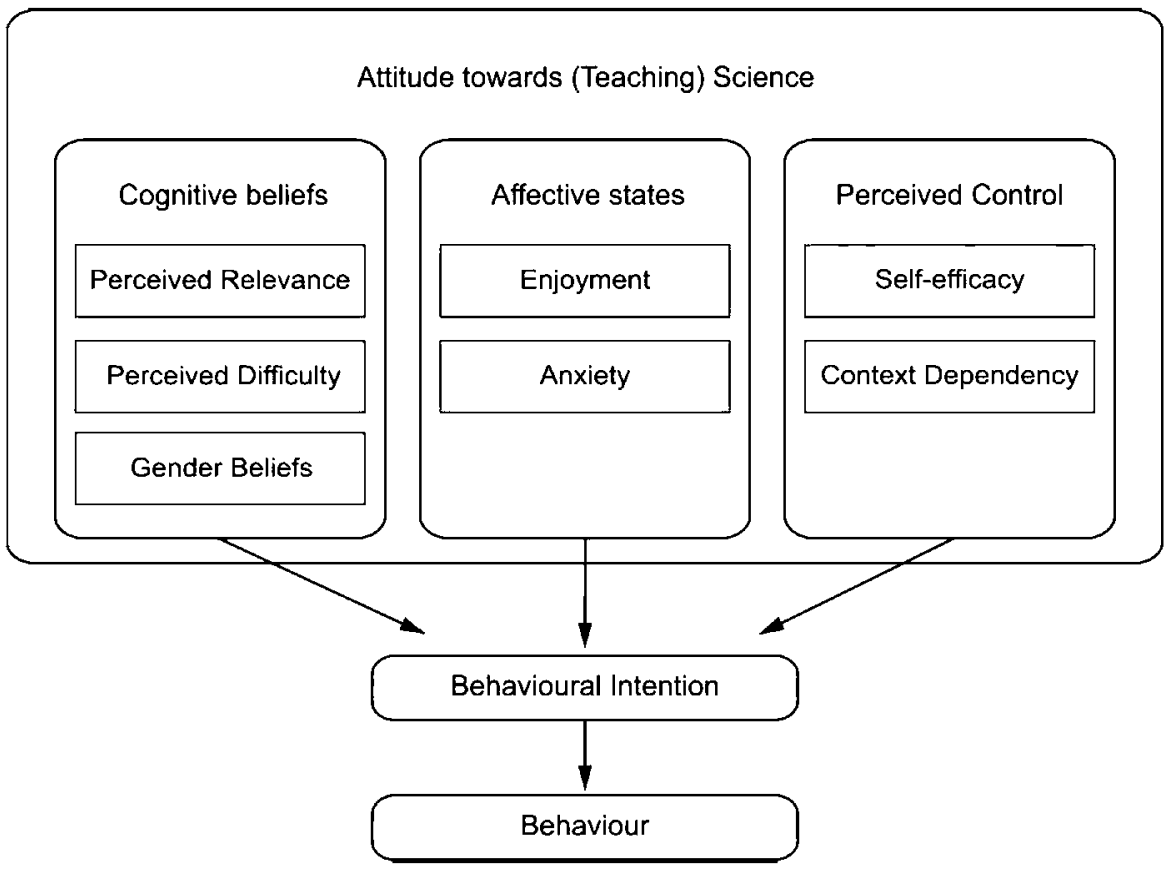

Fuente: van Aalderen-Smeets et al., 2012.

$\mathrm{Al}$ margen de los modelos que intentan dar cuenta de las raíces de las actitudes, se han sucedido estudios empíricos que, como resultados relevantes, apuntan hacia un descenso de las actitudes hacia la Ciencia desde la infancia a la adolescencia y a una relación no clarificada entre actitud y rendimiento académico.

Especialmente interesante resulta la primera evidencia investigadora. Como señalan Vázquez y Manassero (2008), este deterioro de las actitudes en la enseñanza obligatoria no afecta por igual a todas las dimensiones que parecen constituir las actitudes hacia la Ciencia, sino que lo hace de forma especial a la Cien- cia que se enseña en el centro educativo. Haciéndose eco de investigaciones previas, estos autores señalan:

Esta preocupante y progresiva depresión actitudinal hacia la ciencia se atribuye a que la ciencia escolar se va ganando una creciente imagen negativa (autoritaria, aburrida, dificil, irrelevante para la vida diaria y causa de los problemas medio-ambientales que preocupan a la opinión pública) en la mente de los estudiantes. El desarrollo hormonal propio de la adolescencia, que provoca drásticos cambios afectivos, es el caldo de cultivo que fundamenta psicobiológicamente estos y otros cambios observables en la personalidad y la conducta de 
los adolescentes. (Vázquez y Manassero, 2008: 275).

Por otro lado, estos cambios negativos parecen afectar preferentemente a las mujeres y a las disciplinas de Física y Química, y se muestran igualmente dependientes de factores sociales y culturales (Vázquez y Manassero, 2008). A pesar de ello los estudiantes de Educación Secundaria perciben de modo positivo la utilidad de la Ciencia en la vida cotidiana e incluso su existencia como materia del currículo, aunque la primera supera a la segunda, lo que invita a pensar que la Escuela no responde a las expectativas del alumno hacia la Ciencia (Solbes, 2011; Pérez y de Pro, 2013).

En cuanto al papel que el profesora$d o$ pueda desempeñar en el fomento o no de actitudes positivas hacia la Ciencia es variado. Gavidia (2008) establece una serie de recomendaciones a este respecto relacionadas con la elección de los contenidos, la metodología docente, la evaluación de los aspectos axiológicos y la visión que el profesorado mantiene sobre sus estudiantes. Igualmente los propios materiales curriculares usados habitualmente en el aula, como los libros de texto (Solbes, Montserrat y Furió, 2007) no favorecen una adecuada actitud hacia la Ciencia. Estos últimos autores pusieron de manifiesto cómo el profesorado coincidía con los estudiantes en algunas medidas que podrían favorecer una mejor imagen de la Ciencia, aunque admitían no llevarlas a cabo de forma habitual, algo evidenciado ya por Pérez y de Pro (2013). Finalmente señalan otras alternativas para lograr el objetivo de la mejora de esta situación.
En definitiva, dado que en la mayoría de los países es en la Educación Secundaria cuando comienza a fraguarse la orientación vocacional, en forma de itinerarios curriculares que condicionan, en gran medida, las elecciones futuras en los estudios superiores, está claro que las carreras científicas no figuren entre las más elegidas.

Centrándonos más concretamente en las vocaciones científicas, resulta evidente que si, como apuntábamos anteriormente, uno de los factores componentes de las actitudes tiene que ver con la expectativa de éxito, el contemplar las ciencias como difíciles, debe conllevar una pobre actitud y, por ende, una baja prevalencia de elección de aquellas como estudios universitarios. Desde un punto de vista empírico, Vázquez (2013) ha mostrado la estrecha relación entre la intención vocacional y la actitud hacia la Ciencia que se enseña en la escuela. Convert (2005) se pregunta por la razón por la cual, a pesar de que la vitola de dificultad de las materias científicas es ya tradicional, ha sido en las últimas décadas cuando se ha producido con especial virulencia el descenso de las vocaciones científicas en Europa. Para dicho autor, la razón debe estar en la progresiva masificación de la Educación Secundaria, la consiguiente disminución o relajación en los mecanismos de selección de los estudiantes, en las poco halagüeñas expectativas laborales para estos estudios y en la desmitificación de los valores inherentes a la Ciencia y la Tecnología. Estos factores generales, entre otros de índole más particular dependiente de cada Estado (mercado laboral, distri- 
bución geográfica de la oferta educativa...), podrían haber desembocado en la insatisfactoria situación actual de las vocaciones científicas.

El ámbito de las vocaciones científicas también debe verse influido por la imagen que de la Ciencia, del científico y de su trabajo mantengan los alumnos -como componentes intrínsecos de la imagen de la Ciencia-, por cuanto esta representación pueda ser o no atractiva para ellos.

La Ciencia puede verse afectada por visiones negativas de la misma que se presentan entre algunos grupos sociales que la suelen asociar a algunos males crónicos como la belicidad o la contaminación, pero también como contrapuestas a algunos dogmas de índole religioso (Solbes, Montserrat y Furió, 2007). Esta imagen negativa parece afectar más a la Química y, en menor medida, a la Biología.

Con respecto al propio científico, resulta curioso observar cómo durante las décadas de 1930 y 1940 los científicos que aparecían en los medios de comunicación eran hombres blancos, de edad avanzada, que estaban locos o eran malvados. Poco a poco esta imagen ha ido evolucionando, y ahora se muestran como una élite de hombres y mujeres, principalmente de raza blanca, socialmente raros, apolíticos, con bata blanca, gafas y el pelo rizado, obsesionados con su trabajo pero algo distraídos -incluso locos-, mayores, sin familia, y en muchas ocasiones peligrosos (Schibeci, 1986; Whitle, 1997; Avraamidou, 2013).

En cuanto al trabajo científico, según Campanario (1999) los principales estereotipos sobre el mismo se centran sobre: (a) la ausencia de concepciones metafísicas de los científicos (convirtiéndolos en seres racionales que trabajan siempre según la más fría de las lógicas); (b) el uso del método científico sin ninguna interferencia externa tal como la casualidad o la buena suerte; y (c) el mito de la replicación, según el cual una idea científica no es aceptada hasta que no se compruebe experimentalmente por científicos ajenos a la publicación de la misma. Realmente, estas representaciones mentales ayudan poco a que los futuros universitarios sean seducidos para decidir optar por esta clase de profesión.

Por su impacto social, merece una atención especial la divergencia en cuanto al sexo (mujer/hombre) que padecen las vocaciones científicas, aunque no siempre sea sencillo interpretar este sesgo (Vázquez y Manassero, 2009).

En definitiva, los factores motivacionales hacia la Ciencia de los estudiantes de Educación Secundaria encierran distintos matices tanto en cuanto a su propia definición, a las relaciones entre las distintas variables que los conforman, a su dependencia con otros factores internos y externos, a su conexión con la imagen de la Ciencia y de los científicos, así como con sus consecuencias sociales, entre las cuales es el de las vocaciones científicas la que más interesa para el caso que nos ocupa. Por consiguiente, se hace necesario seguir profundizando en la clarificación en las relaciones entre la imagen de la Ciencia (y de su docencia) y las vocaciones científicas, teniendo en cuenta no solo las opiniones de los estudiantes, sino las 
de sus propios profesores. Esto es lo que tratamos de aportar con este trabajo.

Concretando, el objetivo general que enunciamos en la Introducción se traduce en los siguientes objetivos específicos:

1. Detectar los cambios en la imagen de la Física y de su docencia que poseen los alumnos de nuevo ingreso en estudios universitarios de Óptica transcurrido un periodo de cinco años (estudio longitudinal).

2. Analizar la imagen de la Física y de su docencia en función de los estudios universitarios a los que acceden los estudiantes (estudio transversal).

3. Comparar la imagen de la Física de profesores universitarios con las de los estudiantes del mismo nivel educativo (estudio comparativo).

4. Identificar la incidencia de algunas variables personales y sociales en la imagen de la Física y de su docencia (estudio evaluativo).

5. Analizar los cambios en la percepción de los alumnos tras una sesión de experiencias de laboratorio sobre Óptica (estudio descriptivo).

\section{Metodología}

La metodología de investigación empleada ha sido de tipo cuantitativo no experimental, con los estudios particulares señalados en los párrafos anteriores, y basada en todos los casos en el uso del método de encuesta. En la figura II se muestra un organigrama de la investigación.

\section{FIGURA II. \\ Organigrama \\ de la metodología seguida en el trabajo}

Fuente: Elaboración propia

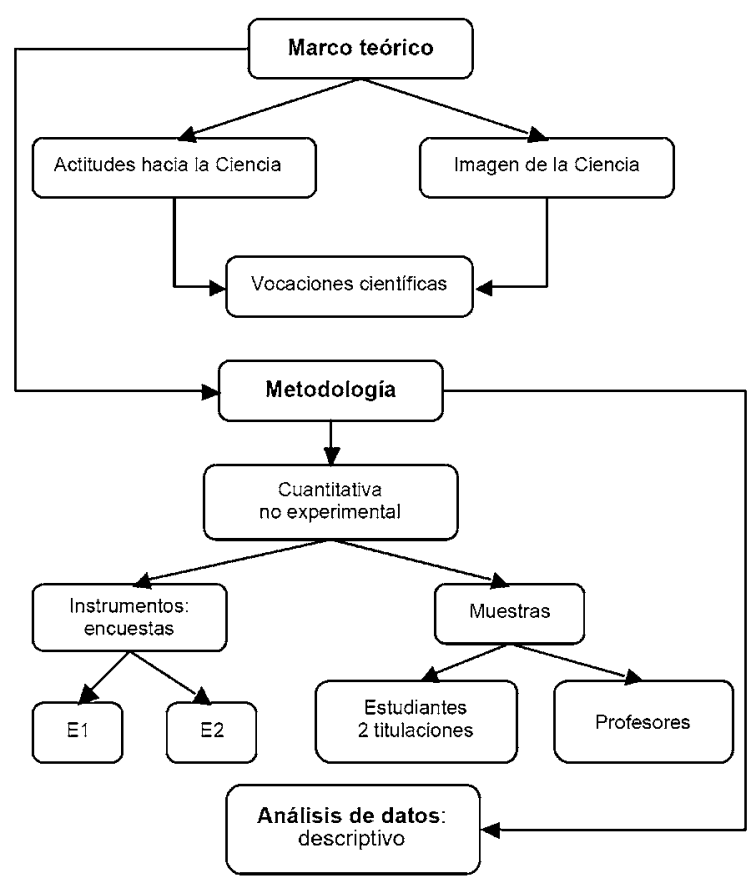


Los instrumentos

Para lograr los objetivos anteriores se diseñaron dos instrumentos tipoencuesta, de carácter anónimo, sobre Física (E1) y sobre Óptica (E2). Para la primera se solicitaban: datos personales, otros relacionados con la docencia de Física recibida (previa y actual) y atribuciones sobre la Física. La segunda se centró exclusivamente en la dis- ciplina de Óptica y se pasó tras haber tenido una sesión de laboratorio con los estudiantes mostrándoles distintas experiencias sobre aplicaciones de la Óptica (Tabla I). En el apartado de Resultados se irán mostrando los ítems de que constaban ambas encuestas. La encuesta $\mathrm{E} 1$ se pasó un mes después del inicio del curso académico y la E2 transcurrido un mes y medio del mismo.

\section{Tabla I. Relación de experiencias mostradas a los alumnos del grupo OA2 (Tabla II).}

\section{Experiencia}

Varilla invisible

Polarización

Fotoelasticidad

Fibra Óptica

Fenómenos de difracción

Anaglifos y hologramas

Simulación Ametropías

Persistencia Visual

Daltónicos

Ilusiones Ópticas

\section{Asignatura de Óptica implicada}

Óptica Geométrica y Óptica Física I

Óptica Física I

Óptica Física I y Tecnología Óptica

Óptica Geométrica y Óptica Física II

Óptica Física II

Visión Binocular, Optometría, Óptica Fisiológica

y Óptica Física II

Óptica Fisiológica y Optometría

Óptica Fisiológica

Óptica Fisiológica

Psicofísica de la Visión

\section{Las muestras}

Las características de las muestras y el año de aplicación de las encuestas se exponen a continuación (Tabla II).

Es de señalar que los grupos OA1 y OA2 proceden del mismo curso de estudiantes, aunque hay ligeras diferencias en su composición por cuanto las encuestas fueron pasadas en días distintos. Si se comparan con el grupo $\mathrm{O}$, es de observar que hay similitud en edad entre ellos aunque hayan pasado 5 años, sin embargo el porcentaje de varones y hembras se ha aproximado en el grupo actual, algo que sí se daba en la muestra de Ingeniería Química. La diferencia de edad media que se aprecia entre Óptica e Ingeniería Química 


\begin{tabular}{|c|c|c|c|c|c|c|}
\hline \multicolumn{7}{|c|}{$\begin{array}{l}\text { Tabla II. Características de las muestras de alumnos encuestadas } \\
\text { (V: varones; M: mujeres). }\end{array}$} \\
\hline $\begin{array}{l}\text { Muestra } \\
(\mathbf{N})\end{array}$ & Encuesta & $\begin{array}{l}\text { Curso de } \\
\text { aplicación }\end{array}$ & Código & Titulación & $\begin{array}{l}\text { Edad } \\
\text { media }\end{array}$ & $\begin{array}{c}\text { Sexo } \\
(\% \mathrm{~V} / \mathrm{M})\end{array}$ \\
\hline 44 & E1 & 2007-08 & $\mathrm{IQ}$ & $\begin{array}{l}\text { Ingeniería } \\
\text { Química }\end{array}$ & 18,4 & $\begin{array}{l}\text { V } 45.5 \\
\text { M } 54.5\end{array}$ \\
\hline 66 & $\mathrm{E} 1$ & 2007-08 & $\mathrm{O}$ & $\begin{array}{l}\text { Óptica y } \\
\text { Optometría }\end{array}$ & 19,6 & $\begin{array}{l}\text { V } 24.2 \\
\text { M } 75.8\end{array}$ \\
\hline 50 & $\mathrm{E} 1$ & $2012-13$ & OA1 & $\begin{array}{l}\text { Óptica y } \\
\text { Optometría }\end{array}$ & 19,4 & $\begin{array}{l}\text { V } 40.0 \\
\text { M } 60.0\end{array}$ \\
\hline 54 & $\mathrm{E} 2$ & $2012-13$ & OA2 & $\begin{array}{l}\text { Óptica y } \\
\text { Optometría }\end{array}$ & 19,4 & $\begin{array}{l}\text { V } 35,2 \\
\text { M } 64,8\end{array}$ \\
\hline 21 & Profesores & $2012-13$ & Prof. & Varias & - & $\begin{array}{l}\text { V } 71.4 \\
\text { M } 28.6\end{array}$ \\
\hline
\end{tabular}

se debe a que en la primera se incorporan algunos alumnos después de haber realizado Formación Profesional o por la vía de mayores de 25 años. Eso no ocurre en Ingeniería Química.

Por otro lado, a algunos de los ítems de la encuesta $\mathrm{E} 1$ respondieron, de forma aleatoria, 21 profesores de tres departamentos de Física de la Universidad de Granada, predominando los pertenecientes al Departamento de Óptica. Respecto a las titulaciones que imparten aquellos predomina Óptica, haciéndolo también en Física, Ingeniería Química, Ingeniería de Telecomunicaciones, Ingeniería Electrónica, Química, Arquitectura y Matemáticas .

Procedimiento de análisis de datos

En concordancia con el tipo de metodología de investigación seguida, se ha centrado en el cálculo de frecuen- cias relativas y medias, diferencia de medias, así como en el recuento de respuestas abiertas.

\section{Resultados}

Encuesta sobre Física (E1)

Iremos exponiendo seguidamente los resultados de cada item.

1. ¿Cursó usted Física en $2^{\circ}$ de Bachillerato?

La Tabla III recoge los datos obtenidos.

\section{Tabla III. Porcentaje de alumnos que cursaron la asignatura de Física en el Bachillerato}

\section{Respuesta}

$$
\text { Grupo }
$$

IQ O OA1

Sí

$\begin{array}{lll}59.1 & 33.3 \quad 30\end{array}$

No 
En Óptica se mantienen los porcentajes en el intervalo de los últimos cinco años. En Ingeniería Química suelen ser más conscientes de que precisan la Física y siguen mayoritariamente la modalidad de Bachillerato de Tecnología; en cambio en el caso de Óptica la consideran como una carrera más propia del itinerario de Ciencias de la Naturaleza y de la Salud, lo que conlleva la obligatoriedad de estudiar Química y Biología, quedando la Física como materia optativa en el segundo curso. Eso puede explicar un déficit en la base física entre los estudiantes de Óptica de primer año universitario.

2. En caso negativo, ¿̇por qué no lo hizo?

Las respuestas más frecuentes, en las dos aplicaciones de la encuesta, son:

- "O elegía Química y Biología, o Física y Dibujo, y prefería la primera opción".

- "En mi centro no la ofertaban".

- "No sabía qué iba a estudiar".

- "No sabía que me hacía falta para Óptica".

$\mathrm{Al}$ margen de la segunda respuesta, el resto indican una baja preferencia hacia la Física o una desorientación vocacional.

3. ¿Ha realizado prácticas de laboratorio en el Bachillerato?

Las respuestas se recogen en la Tabla IV.

Llama la atención, en todos los casos, el bajo porcentaje de alumnos que realizaron en el Bachillerato prácticas de laboratorio que, inclusive, disminuyen para los que cursaron Óptica a lo largo de los últimos cinco años. Estos resulta- dos confirman los de otros autores (Solbes, Montserrat y Furió, 2007; Pérez y de Pro, 2012). Cabría preguntarse por la imagen que de la Física (y de la Ciencia en general) pueden tener unos alumnos que no han "pisado" un laboratorio.

\section{Tabla IV. Porcentaje de alumnos que realizaron prácticas de laboratorio en el Bachillerato}

Respuesta

\begin{tabular}{lccc} 
& IQ & O & OA1 \\
\hline Sí & 18.2 & 28.8 & 16 \\
No & 81.8 & 71.2 & 84 \\
\hline
\end{tabular}

4. ¿Su profesor le explicaba la Física haciendo uso de experiencias de cátedra?

Se exponen los resultados en la Tabla V.

\section{Tabla V. Porcentaje de alumnos a los que sus profesores mostraban experiencias de cátedra en el Bachillerato}

\begin{tabular}{lccc}
$\begin{array}{l}\text { Frecuencia } \\
\text { de uso }\end{array}$ & \multicolumn{3}{c}{ Grupo } \\
\hline $\mathbf{1}$ (Nunca) & 22.7 & 43.9 & 42.0 \\
$\mathbf{2}$ & 15.9 & 22.7 & 16.0 \\
$\mathbf{3}$ & 20.5 & 24.2 & 24.0 \\
$\mathbf{4}$ (Siempre) & 15.5 & 4.6 & 14.0 \\
$\mathbf{5}$ (Sie & 4.0 \\
\hline
\end{tabular}

Estos resultados corroboran los anteriores, ya que incluso las experiencias demostrativas suelen ser infrecuentes, afectando más a los alumnos de Óptica que a los de Ingeniería Química. En el caso de Óptica, esta tendencia se ha 
visto alterada durante los últimos cinco años, en los que parece haber mejorado algo el porcentaje de uso.

5. ¿Su profesor le explicaba la Física haciendo uso constante de ejemplos de la vida cotidiana?

En la Tabla VI se representan los resultados al anterior item.

Tabla VI. Porcentaje de alumnos a los que sus profesores de Bachillerato explicaban la Física mediante ejemplos de la vida cotidiana

\begin{tabular}{lccc}
$\begin{array}{l}\text { Frecuencia } \\
\text { de uso }\end{array}$ & \multicolumn{3}{c}{ Grupo } \\
\hline $\mathbf{1}$ (Nunca) & 18.2 & 22.7 & 28.0 \\
$\mathbf{2}$ & 20.5 & 22.7 & 26.0 \\
$\mathbf{3}$ & 18.2 & 22.7 & 12.0 \\
$\mathbf{4}$ (Siempre) & 22.7 & 25.8 & 26.0 \\
$\mathbf{5}$ (Sie.5 & 6.1 & 8.0 \\
\hline
\end{tabular}

Las actividades cotidianas -externas a las actividades escolares- que implican de algún modo a la Ciencia constituyen la base de una construcción social de la misma (Zimemerman y Bell, 2012), por lo que su inserción en la explicaciones de aula son fundamentales para adquirir una correcta imagen de la Ciencia (Gavidia, 2008). La Tabla $\mathrm{V}$ nos muestra un mayor porcentaje de uso en los alumnos de Ingeniería Química (seguramente por haber cursado Física en mayor proporción que los de Óptica). Tampoco se aprecia un cambio de tendencia muy significativo para los alumnos de Óptica en los últimos cinco años, aunque aumentan los alumnos a los que nunca o casi nunca se les ense- ñó Física haciendo uso de ejemplos de la vida cotidiana. Una hipótesis plausible es que durante el segundo año de Bachillerato la Física se orienta más hacia el examen de Selectividad que a conseguir que los alumnos la vinculen con su entorno.

6. ¿Ha visitado el Parque de las Ciencias? ¿Cómo calificaría la visita entre 1 (poco interesante) y 5 (muy interesante)?

Los museos de Ciencia y otras instalaciones similares han ido creciendo en nuestro país, entre otras razones, porque han contado con el favor popular. De hecho, el Parque de las Ciencias de Granada es hoy día el museo más visitado de Andalucía. Ello representa una prueba fehaciente de que la Sociedad, en general, se interesa por la Ciencia, aunque, como ya mencionamos con anterioridad, la que se enseña en nuestras aulas no suele conectar con ese interés general. En la Tabla VII se muestran los resultados a la cuestión anterior.

Tabla VII. Porcentaje de alumnos que han visitado (o no) el Parque

de las Ciencias de Granada y calificación media de la misma (de 1 a 5)

\begin{tabular}{lccc} 
Respuesta & \multicolumn{3}{c}{ Grupo } \\
& IQ & O & OA1 \\
\hline Sí & 84.1 & 78.4 & 84 \\
No & 15.9 & 21.6 & 16 \\
Calificación & 4.36 & 4.18 & 4.45 \\
\hline
\end{tabular}

Como se aprecia, un gran porcentaje de los alumnos ha visitado el Parque de las Ciencias (los que no lo han hecho tie- 
nen su residencia habitual fuera de la ciudad) y lo valoran muy positivamente. Existen pocas variaciones entre grupos, aunque sí un ligero aumento en frecuencia de las visitas y calificación de las mismas durante los últimos cinco años.

7. Marque cuatro de los siguientes adjetivos de acuerdo a lo que usted piensa de la Física.

Con este item se pretendía que, tanto los estudiantes como los profesores participantes (a estos últimos se les pedía que señalaran cómo les gustaría que sus alumnos percibieran la Física), pudieran seleccionar algunos atributos de índole afectiva y cognitiva mediante los que calificar la Física, lo que sin duda podría acercarnos a perfilar la imagen de Física que poseen. Los resultados obtenidos aparecen en la Tabla VIII.

Los resultados obtenidos merecen algunas consideraciones:

- En general hay una gran coincidencia entre los tres grupos de alumnos, con pequeñas diferencia entre los de Óptica y los de Ingeniería.

- Respecto a las modificaciones temporales (grupos O y OA1), se mantienen las características generales, si bien mejora la consideración en adjetivos como "Entretenida", "Muy numérica-Fenomenológica", "Útil" y "Práctica". Un análisis más detallado de estos datos se muestra en la Tabla IX. Quizás pareciera que algunas iniciativas públicas de divulgación científica que van proliferando (Semanas de Ciencia, algunos programas de TV...) han hecho que mejore la imagen de la Física en cuanto a calificarla como
Tabla VIII. Porcentajes de respuesta de las muestras de estudiantes y profesores a las opciones que se les mostraban sobre la Física

\begin{tabular}{lcccc}
\hline Atributo & \multicolumn{4}{c}{ Muestras } \\
& IQ & 0 & OA1 & Profes. \\
\hline Difícil & 79.5 & 75.8 & 76 & 0 \\
Fácil & 0 & 4.5 & 0 & 14.3 \\
Bonita & 13.6 & 10.6 & 8 & 61.9 \\
Fea & 4.5 & 15.2 & 2 & 0 \\
Entretenida & 38.6 & 37.9 & 48 & 52.4 \\
Aburrida & 13.6 & 7.6 & 4 & 0 \\
Abstracta & 43.2 & 39.4 & 24 & 4.8 \\
Tangible & 11.4 & 9.1 & 8 & 52.4 \\
Muy numérica & 20.5 & 60.6 & 32 & 0 \\
Fenomenológica & 22.7 & 13.6 & 20 & 66.7 \\
Útil & 65.9 & 43.9 & 54 & 81.0 \\
Inútil & 0 & 4.5 & 0 & 0 \\
Práctica & 70.4 & 59.1 & 78 & 47.6 \\
Teórica & 11.4 & 4.5 & 14 & 19.1 \\
\hline
\end{tabular}

entretenida o útil, lo que está en sintonía con el estudio de Pérez y de Pro (2013).

- De los 14 adjetivos que se proponían, los tres en los que aparece una mayor diferencia son "Muy numérica, útil y práctica”. Los ingenieros consideran la física más práctica y útil y menos numérica que los alumnos de Óptica, lo que coincide con nuestras percepciones sobre ambos grupos de alumnos. Los ingenieros reconocen que es práctica y útil, aunque siguen teniendo una mala imagen de la física; los ópticos no tienen muy claro para qué les sirve esta asignatura por lo que la utilidad que le dan es menor. 
- Cabría preguntarse si existe una relación entre la imagen de la física y la vocación de los estudiantes. La imagen que tienen ambos grupos es muy similar, con las pequeñas diferencias antes comentadas, lo que, sin duda, es ciertamente llamativo puesto que cabría esperar que aquellos estudiantes con una vocación más científica y tecnológica tuvieran una mejor imagen de la física que aquellos otros que tienen una vocación más sanitaria. Evidentemente, esto debemos considerarlo como un indicio ya que sólo hemos analizado dos grupos de alumnos diferentes, pero, sin duda, este indicio merecería una profundización en trabajos posteriores.

- Por otra parte, quizás la diferencia más significativa entre los dos grupos radique en haber cursado o no Física en $2^{\circ}$ de Bachillerato (Tabla III), ya que en el grupo de Ingeniería es mayor el porcentaje que la cursó, por lo que, por así decirlo, sería de esperar que ese año extra hubiera servido para mejorar la imagen que tuvieran de ella; sin embargo, esto no ocurre, podríamos suponer que los alumnos de Ingeniería la cursan en $2^{\circ}$ de Bachillerato porque es necesario para abordar los estudios de ingeniería, pero que ello no les ha hecho mejorar la imagen que de ella mantienen.

- Es patente la gran discrepancia entre los resultados anteriores y lo que expresan los profesores sobre cómo les gustaría que percibieran la Física los estudiantes. Por ejemplo, difícil: $75 \%$ los alumnos y $0 \%$ los profesores; bonita: $10 \%$ y $62 \%$, respectivamente; y así con tangible, fenomenológica...

Parece que la contradicción está servida: los profesores deseamos que los estudiantes contemplen la Física de una forma, pero conseguimos justo lo contrario.

En la Tabla IX se establece una comparación por parejas de atributos antónimos para los dos grupos de Óptica y el de profesores.

Parece ser, de los datos de la Tabla IX, que tras cinco años, seis de las siete pare-

\section{Tabla IX. Diferencias de porcentajes (redondeados) para los atributos antónimos en los grupos O, OA1 y Profesores}

\begin{tabular}{lrrr}
\hline Atributos & \multicolumn{1}{c}{ 0 } & \multicolumn{1}{c}{ Grupos } \\
& \multicolumn{1}{c}{ OA1 } & \multicolumn{1}{c}{ Profesores } \\
\hline Difícil/Fácil & $75.8-4.5=+71$ & $76-0.0=+76$ & $0.0-14.3=-14$ \\
Bonita/Fea & $10.6-15.2=-5$ & $8.0-2.0=+6$ & $61.9-0.0=+62$ \\
Entretenida/Aburrida & $37.9-7.6=+30$ & $48.0-4.0=+44$ & $52.4-0.0=+52$ \\
Abstracta/Tangible & $39.4-9.1=+30$ & $24.0-8.0=+16$ & $4.8-52.4=-48$ \\
Muy Numérica/Fenomenológica & $60.6-13.6=+47$ & $32.0-20.0=+12$ & $0.0-66.7=-67$ \\
Útil/Inútil & $43.9-4.5=+39$ & $54.0-0.0=+54$ & $80.9-0.0=+81$ \\
Práctica/Teórica & $59.1-4.5=+55$ & $78.0-14.0=+54$ & $47.6-19.0=+29$
\end{tabular}


jas de atributos se aproximan, aunque sea levemente, a la opinión de los profesores y empeora en la séptima (difícil/fácil).

Pero, ¿por qué les parece a los estudiantes difícil la Física? ¿Por los conceptos físicos o por las matemáticas que usa?

8. Marque con un 1 (nada de acuerdo) hasta un 5 (totalmente de acuerdo) cada una de las siguientes afirmaciones.
Mediante este item se pretendía poner de manifiesto el dilema físicamatemáticas como fuente principal de dificultades para los estudiantes. En la Tabla X se muestran las puntuaciones medias asignadas a cada una de las afirmaciones.

Los grupos encuestados hace cinco años atribuyen más dificultad a las

\section{Tabla X. Puntuaciones medias que asignan los grupos de estudiantes a las afirmaciones que se les proporcionan}

\begin{tabular}{lccc}
\hline Afirmación & \multicolumn{3}{c}{ Grupos } \\
& IQ & O & OA1 \\
\hline Entiendo los conceptos, pero me pierdo en las matemáticas que usa & 2.98 & 3.83 & 2.58 \\
Me cuesta mucho entender los conceptos físicos pues son muy abstractos & 2.53 & 3.11 & 2.9 \\
\hline
\end{tabular}

Matemáticas que a la propia Física, y en mayor proporción los de Óptica que los de Ingeniería. Transcurrido el periodo de tiempo señalado, parece haber un intercambio de papeles, y ahora se muestra más difícil la Física que las Matemáticas; quizás esto sea síntoma de que se emplean menos problemas de resolución matemática compleja en Educación Secundaria.
9. A priori, sobre la asignatura de Física que acaba de empezar, valore de 1 (nada de acuerdo) hasta 5 (totalmente de acuerdo) las siguientes afirmaciones.

Este item tenía una estructura similar al anterior pero ahora está dedicado a las predicciones y opiniones que hacen los estudiantes respecto a la asignatura de Física que están iniciando. Los resultados se presentan en la Tabla XI.

\section{Tabla XI. Puntuaciones medias que asignan los grupos de estudiantes a las afirmaciones respecto de la Física que se les proporcionan}

\begin{tabular}{lccc}
\hline Afirmación & \multicolumn{3}{c}{ Grupos } \\
& IQ & O & OA1 \\
\hline Creo que va a ser muy difícil & 4.25 & 3.89 & 3.50 \\
Las prácticas me van a ayudar a mejorar en muchos aspectos & 3.11 & 3.62 & 3.48 \\
En la carrera que acabo de empezar creo que la física es muy importante & 3.88 & 4.09 & 4.38 \\
Para mi futuro profesional la formación en física me aportará mucho & 3.43 & 3.37 & 3.66
\end{tabular}


Un somero análisis de los datos anteriores nos viene a decir que, en general, los alumnos piensan que la asignatura de Física es difícil e importante y, en menor grado, que las prácticas de laboratorio pueden ayudarles a superar las dificultades y que les va a servir para su futuro profesional. El cómputo global es más negativo para los ingenieros que para los ópticos. Respecto a los cambios entre los grupos $\mathrm{O}$ y OA1, ha disminuido la percepción de dificultad y la confianza en que las prácticas le ayuden; por el contrario aumenta la importancia que se le concede a la Física.
10. Indique lo que debería hacerse (tanto por su parte como por parte del profesor) para mejorar su rendimiento en Física.

Con este item se pretendía que los alumnos hicieran propuestas concretas, tanto respecto a ellos mismos como a sus profesores, para mejorar su rendimiento académico en Física. Dado que había un transcurrido en torno a un mes de clases, estimamos que era ya un tiempo suficiente para considerar fiables estos datos. En la Tabla XII se han ordenado por frecuencias las respuestas de los alumnos de los diferentes grupos.

\section{Tabla XII. Sugerencias de los estudiantes para mejorar su rendimiento en Física ordenadas por frecuencias}

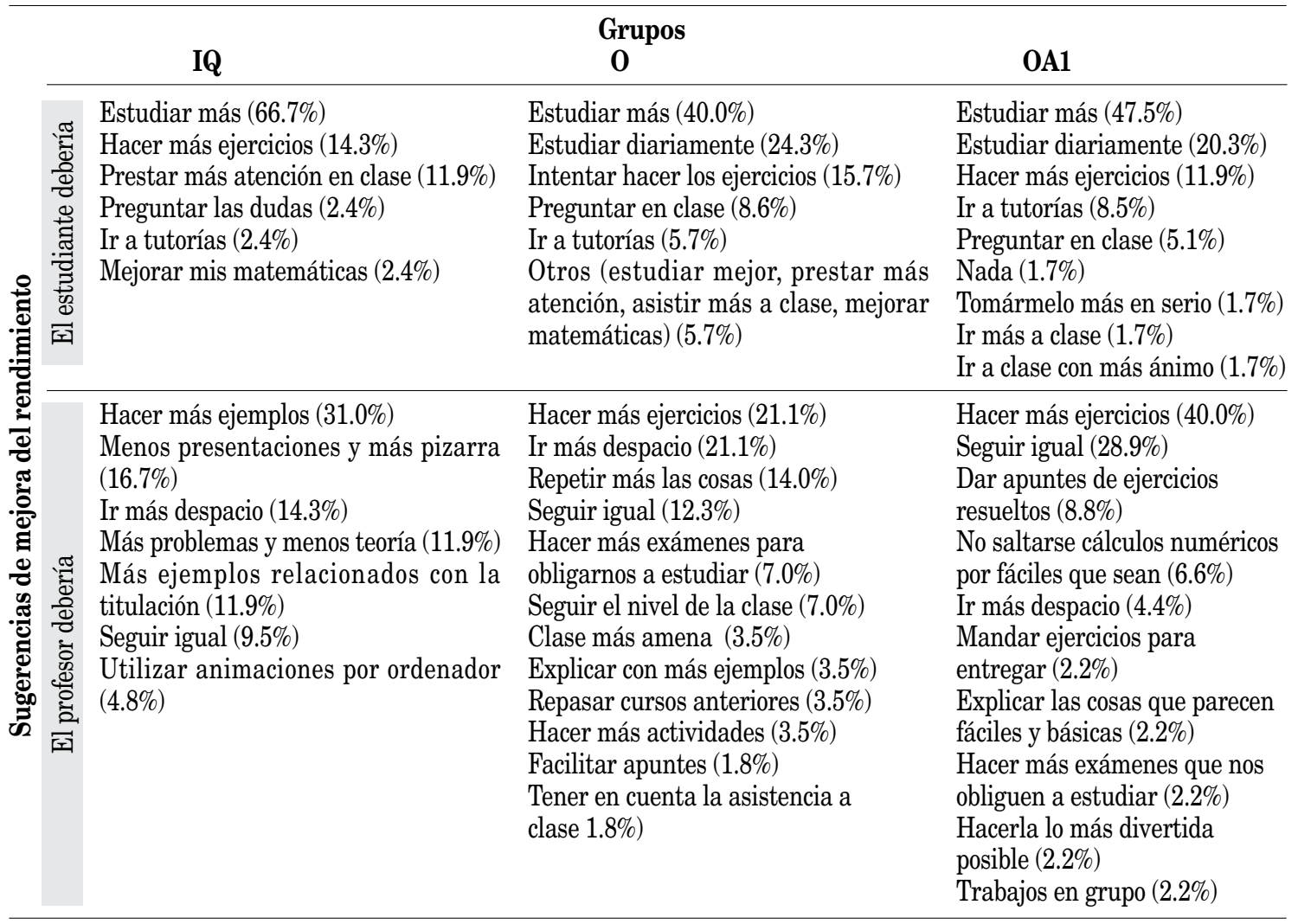


Si tomamos en consideración las respuestas con una frecuencia mayor del $5 \%$, en general encontramos con respecto a los propios estudiantes que para mejorar su rendimiento deberían:

- Estudiar más tiempo y con continuidad.

- Resolver más problemas o intentarlo.

- Atender e intervenir más en clase.

- Hacer uso de las tutorías.

No se aprecian diferencias relevantes entre los grupos de estudiantes encuestados.

En relación con lo que deberían hacer sus profesores, las respuestas presentan una mayor diversidad, destacando las siguientes propuestas (entre paréntesis se señalan las respuestas menos frecuentes dentro de cada categoría y sugeridas por uno solo de los grupos):

- Resolver más problemas (y menos teoría).

- Poner más ejemplos (relacionados con la titulación).

- Ralentizar el ritmo de las clases (y repetir algunas cosas o cálculos).

- Seguir igual.

- Otros (utilizar más la pizarra y menos las presentaciones, hacer más exámenes, distribuir problemas resueltos, adaptarse al nivel de la clase).

Con algunas variaciones, las respuestas intergrupos responden a un patrón que reclama una enseñanza más aplicada, centrada en problemas y con un ritmo más lento, aunque también con un significativo respaldo a como se les viene enseñando.

Como nexo común en lo que deberían hacer alumnos y profesores, se halla la preocupación por la resolución de problemas, seguramente porque constituyen un componente esencial en la evaluación de la asignatura.

\section{Encuesta sobre Óptica (E2)}

Como se comentó con anterioridad, esta encuesta se pasó a un grupo de alumnos de Óptica y Optometría (OA2), perteneciente al mismo curso que OA1, aunque con ciertas variaciones en cuanto a los alumnos que respondieron, ya que se hizo en una fecha diferente a la E1.

Veamos los ítems y las respuestas obtenidas.

1. ¿Por qué me matriculé en el Grado de Optica y Optometría? Marca, de las siguientes afirmaciones, las que más se aproximen a las razones que te llevaron a matricularte (como máximo 3).

Con este item se pretendía preguntar directamente por las razones de la elección de carrera por los estudiantes. Las respuestas se muestran en la Tabla XIII.

Como se deduce de las respuestas, los alumnos se dejan guiar principalmente por el futuro profesional, por razones familiares y por la orientación vocacional recibida, sin olvidar que un número apreciable de ellos no eligió estos estudios en primera opción. Solo algunas respuestas agrupadas en el epígrafe de "otras" parecen responder a una verdadera vocación.

2. Conocimiento que tenía de la Titulación y cómo te encuentras en ella. Valórelas según su grado de acuerdo con ellas, desde 1 (nada) hasta 5 (totalmente).

En este item se intenta profundizar más en las razones anteriores y en sus 


\section{Tabla XIII. Razones por las que se matricularon los alumnos del grupo OA2 en la carrera de Óptica y Optometría \\ (ordenadas de mayor a menor frecuencia)}

Razones

Me habían dicho que tiene buenas salidas profesionales

No pude entrar en lo que quería y ésta es una segunda opción

Fui a unas charlas de orientación y me gustó lo que dijeron

Me lo aconsejaron mis padres

Mi familia tiene una Óptica y me gusta el trabajo

He hecho el módulo de anteojería y quería ampliar estudios

Me lo aconsejaron en el instituto

Otras (fundamentalmente "me gusta")

\section{Tabla XIV. Conocimiento y motivación de los estudiantes del grupo OA2 hacia los nuevos estudios (Exp. Sí: realizaron la experiencia del item 3; Exp. No: no la realizaron)}

\section{Afirmación}

Exp. Sí Exp. No Todos

Conocimiento que tenía de las asignaturas que la constituyen

3.46

3.07

3.35

Conocimiento que tenía de los contenidos

2.74

2.47

2.67

Grado de motivación que tenía

3.73

4.16

Cada vez estoy más motivado/a para estudiarla

4.03

3.27

3.82

Creo que acerté plenamente cuando me matriculé en este grado

primeras impresiones tras comenzar sus estudios universitarios.

Las respuestas (Tabla XIV) se han separado entre los alumnos que asistieron a la sesión de experiencias -Exp. Sí- (véase el próximo item), los que no lo hicieron -Exp. No- y el total de alumnos.

Comentaremos estos resultados en el siguiente ítem.

3. Con respecto a la sesión realizada en el laboratorio, por favor, valore desde 1 (nada de acuerdo) hasta 5 (totalmente de acuerdo) las siguientes afirmaciones.
Como ya se comentó en el apartado de Metodología, transcurrido en torno a mes y medio del inicio del curso, los estudiantes pudieron asistir voluntariamente a una sesión de experiencias de laboratorio sobre distintos aspectos de la Óptica (Tabla I). Los resultados del item de valoración de la misma se muestran en la Tabla XV.

De la Tabla XIV y, sin ánimo de establecer relaciones causa-efecto, parece claro que los alumnos que asistieron voluntariamente a la sesión de labora- 


\section{Tabla XV. Valoración de la sesión de laboratorio sobre Óptica que recibieron los alumnos del grupo OA2.}

Afirmación

Frecuencia

media

Casi todas la cosas que vimos eran desconocidas para mí

3.08

Me pareció interesante que en los próximos cursos vayamos a estudiar cosas

como las que vimos

4.46

Me ayudó a conocer el Grado en el que estoy

4.23

Fue una experiencia simpática y divertida

4.69

Si hubiera sabido cómo iba a ser, no habría venido a clase

La experiencia ha contribuido a que esté más satisfecho/a de haberme matriculado en Óptica

Fue una experiencia adecuada para un día como en el que fue, pero nada más

Me ha servido para darme cuenta de que la Óptica es mucho más de lo que yo creía

Creo que me sirvió para motivarme más para estudiar

Creo que de vez en cuando deberíamos hacer actividades que nos acerquen a lo que veremos otros años

Creo que, aunque estemos en $1^{\circ}$, de vez en cuando deberíamos hacer actividades que nos acerquen a nuestro futuro profesional

Esta experiencia ha cambiado mi percepción sobre las posibilidades de estos estudios

torio poseen una motivación intrínseca hacia los estudios de Óptica significativamente superior a la de los que no asistieron.

Los datos de la Tabla XV admiten diversas lecturas, aunque centrándonos en las puntuaciones exclusivamente, observamos cómo la experiencia fue percibida como positiva de forma global. Al margen de la respuesta emocional ("Fue una experiencia simpática y divertida"), se aprecia que lo más valorado tiene que ver con la orientación que supuso respecto a los estudios que están realizando. A continuación se sitúan los ítems relacionados con un cambio cognitivo y afectivo en la percepción sobre la Óptica. El resto de las puntuaciones obtenidas en los ítems que minusvaloraban la experiencia refuerzan lo significativa que para los estudiantes fue dicha experiencia.

Para esta encuesta y muestra de alumnos se realizaron unos análisis de datos complementarios mediante el paquete estadístico SPSS (test de contraste de diferencias de medias) con el fin de dilucidar la influencia de algunas de las variables consideradas. Es de resaltar que, dado el tamaño de la muestra, para que exista significación estadística, las diferencias en las medias han de ser considerables.

Sexo

Aparecen diferencias estadísticamente significativas en la afirmación 
(Tabla XIII) "no pude entrar en lo que quería y esta es mi segunda opción" $(\mathrm{p}=0.025)$ y diferencias bastante apreciables aunque no con ese nivel de significación en (Tabla XV): "Creo que de vez en cuando deberíamos hacer actividades que nos acerquen a lo que veremos otros años" $(\mathrm{p}=0.089)$, a favor de las mujeres en ambos casos. Parece confirmarse que éstas presentan una menor predisposición hacia los estudios de ciencias (Solbes, Montserrat y Furió, 2007), pero aprecian el valor de actividades como la realizada para invertir esa tendencia.

\section{Edad}

Diferencias significativas en (Tabla XIII): "He hecho el módulo de anteojería y quería ampliar estudios" ( $\mathrm{p}=0.000)$; en este caso, lógicamente, a favor de los alumnos de mayor edad.

\section{Realización de la Experiencia}

Se aprecia diferencia en cuanto a la afirmación en (Tabla XIV): "Creo que acerté plenamente cuando me matriculé en este grado" ( $\mathrm{p}=0.090)$, a favor de los que realizaron la Experiencia.

Si combinamos más de una variable, el número de individuos se reduce y, por tanto, resulta más difícil alcanzar una significación estadística, aún así resaltamos la siguiente:

Combinación de sexo, edad y realización de la Experiencia

(Tabla XIV) "Conocimiento que tenía de los contenidos" $(\mathrm{p}=0.069)$.

De los datos anteriores (junto con otros que no hemos incluido por falta de significación estadística) parece desprenderse que:
- Los alumnos que proceden de formación profesional ("realizaron el módulo de anteojería”) son de mayor edad, conocían menos los contenidos de la titulación, pero están más motivados, quieren hacer las actividades y están contentos con haberse matriculado, aunque para ellos todo es mucho más difícil (se encuentran con grandes deficiencias en Matemáticas y Física). Parece que la información que reciben en Formación Profesional Ocupacional sobre ambas materias es más escasa.

- Algunos alumnos que acceden desde el Bachillerato querrían haber realizado otros estudios y conocían mejor lo que se iban a encontrar.

- La mujeres son más entusiastas y participativas, los hombres menos.

\section{Conclusiones}

En este artículo hemos pretendido hacer algunas aportaciones a la imagen de la Física universitaria, de su enseñanza y de la actitud de los alumnos ingresantes. Para ello hemos diseñado dos encuestas, una de ellas repetida en un intervalo de cinco años y hemos contado también con la opinión de profesores universitarios de Física. Las muestras participantes no son intencionales por lo que no pretenden ser representativas de la población y por lo tanto no cabe establecer generalizaciones, pero sí pensamos que los resultados pueden aportarnos algunas reflexiones acerca del preocupante descenso de vocaciones 
científicas, común en la mayoría de los países desarrollados.

Algunos de los resultados validan el modelo denominado teoría de la expectativa-valor (figura I) y, por otro lado, muestran el escaso seguimiento de las recomendaciones efectuadas por los tres informes citados en la Introducción de este trabajo.

Vamos a ir relacionando las conclusiones con los objetivos que nos planteamos:

1. Detectar los cambios en la imagen de la Física y de su docencia que poseen los alumnos de nuevo ingreso en estudios universitarios de Óptica transcurrido un periodo de cinco años.

Los cambios más notables en las respuestas de los estudiantes en este intervalo de tiempo serían:

(a) Disminuye el porcentaje de alumnos que realizaron en el Bachillerato prácticas de laboratorio, aunque aumenta ligeramente el de experiencias de cátedra.

(b) Aumenta el porcentaje de alumnos a los que nunca o casi nunca se les enseñó Física con ejemplos de la vida cotidiana.

(c) Aumenta el porcentaje de los que han visitado el "Parque de las Ciencias" de Granada y su valoración de esa visita.

(d) Aumenta la consideración positiva de la Física, aproximándose a la que manifiestan los profesores.

(e) El grupo actual percibe mayor dificultad en la Física que en las Matemáticas, lo contrario de lo que ocurría en el grupo de hace cinco años.

(f) Existe una disminución en la percepción de dificultad de la Física y en la confianza de que las prácticas de laboratorio les ayuden; por otra parte aumenta la importancia que se le concede a la Física.

Una valoración global de los resultados indica algunos signos de mejora hacia la imagen de la Física, a pesar de que no se permite vislumbrar el mismo cambio de rumbo en la docencia recibida en Bachillerato.

2. Analizar la imagen de la Física y de su docencia en función de los estudios universitarios a los que acceden los estudiantes.

De la comparación de resultados entre los grupos de Ingeniería y de Óptica (IQ y O), entrevistados hace cinco años, podemos extraer algunas conclusiones:

(a) Los alumnos de IQ han elegido en mayor proporción la modalidad de Bachillerato de Tecnología.

(b) Los alumnos de $\mathrm{O}$ parecen haber realizado más prácticas de laboratorio en Bachillerato; por el contrario recibieron menos experiencias de cátedra.

(c) Los alumnos de IQ han visitado con mayor frecuencia el "Parque de las Ciencias".

(d) En cuanto a los atributos de la Física, la diferencia más notable está en que los estudiantes de $\mathrm{O}$ consideran muy numérica la Física, frente a los de IQ que la estiman más fenomenológica.

(e) Los alumnos de IQ perciben menos dificultad en los conceptos físicos y en las matemáticas auxiliares, y confían menos en que las prácticas les puedan ayudar a superar esas dificultades.

De forma general, parece que los alumnos de IQ se han preparado desde 
el Bachillerato para estudiar Física, por lo que se sienten más seguros de sí mismos y motivados hacia esta disciplina. En otro orden de cosas parece que la frecuencia de las prácticas de laboratorio y de cátedra se relacionan de forma inversamente proporcional.

3. Comparar la imagen de la Física de profesores universitarios con las de los estudiantes del mismo nivel educativo.

No resulta frecuente contrastar lo que piensan los profesores sobre la materia que enseñan y lo que piensan los alumnos. En nuestro caso, se ha puesto de manifiesto una visión casi contrapuesta, que reitera lo difícil que puede resultar llegar a un punto de confluencia que haga más factible ajustar lo que los profesores pensamos y lo que nuestros alumnos perciben. Esto es sin duda una condición sine qua non para comenzar a cambiar la docencia.

En cuanto a esta última observación hemos de reconocer que cuando se requiere de los alumnos que planteen propuestas de mejora, las ejercen con responsabilidad, ya que las reparten equitativamente entre ellos mismos y sus profesores. No obstante, en ningún caso se plantean una "revolución" en la forma de enseñar y aprender sino que mayoritariamente reinciden en un modelo aproximadamente tradicional: estudiar más, preguntar en clase, resolver más problemas, que el profesor lleve un ritmo más lento... Seguramente carecen de referentes para pensar en que "otra forma de enseñar es posible".

4. Identificar la incidencia de algunas variables personales y sociales en la imagen de la Física y de su docencia.
Nos planteamos profundizar en la posible influencia de algunas variables de la muestra que respondió a la segunda encuesta. Ello nos permitió detectar perfiles diferenciados de las respuestas en función de los estudios de procedencia, edad y sexo, fundamentalmente. Estos resultados nos deberían obligar a atender a esa diversidad y proponer un tratamiento diferenciado en función de sus intereses y conocimientos previos, que busque "enganchar" a los estudiantes que perciben la Física como un muro infranqueable.

5. Analizar los cambios en la percepción de los alumnos tras una sesión de experiencias de laboratorio sobre Ópti$c a$.

La experiencia de llevar a los alumnos al laboratorio de Óptica y mostrarles algunas situaciones y dispositivos anticipatorios de las posibilidades que sus estudios ofrecen, tanto respecto de fenómenos como de aplicaciones, parece que se muestra como una alternativa para reorientar vocacionalmente a los estudiantes noveles, máxime si se tiene en cuenta que un número relevante de ellos no han ingresado por razones altruistas y afectivas.

Estos últimos comentarios nos permiten enlazar con la búsqueda de alternativas a la situación reflejada en este trabajo, reforzando la vinculación Universidad y Secundaria mediante distintas iniciativas como las realizadas por el Grupo de Divulgación de la Óptica de la Universidad de Granada, que incluyen la oferta de visitas al Laboratorio de Óptica y presencia en el "Parque de las Ciencias" con iniciativas puntuales 
o exposiciones temporales (García et al., 2010a; 2010b; 2012).

Tampoco debemos olvidar una problemática siempre presente entre los estudiantes cuando se enfrentan a asignaturas no directamente relacionadas con el perfil profesional de sus estudios, en nuestro caso, "Física para no físicos", en cuyo caso parecen no verla importante, no la perciben como materia fundamental. A ello tampoco ayuda la endogámica tendencia española cuando se reforman las enseñanzas universitarias, en donde los títulos se cierran cada vez más sobre sí mismos, excluyendo a otras materias (y departamentos) considerados como "foráneos", así como la falta de preparación de algunos profesores que no adaptan los currículos de sus asignaturas al perfil profesional de las titulaciones (Física para ingenieros, Física para biólogos...).

\section{Bibliografía}

AVRAAMIDOU, L. (2013), Superheroes and supervillains: reconstructing the mad-scientist stereotype in school science, Research in Science and Technological Education, vol. 31, pp. 1-25. DOI: 10.1080/02635143.2012.761605

CAMPANARIO, J. M. (1999), La ciencia que no enseñamos, Enseñanza de las Ciencias, vol. 17, pp. 397-410.

COMISIÓN EUROPEA (2011), Eurydice. Science in Europe:National Policies, Practices and Research, Bruselas, Audiovisual and Culture Executive Agency. http://eacea. ec.europa.eu/education/eurydice
CONVERT, B. (2005), Europa y la crisis de las vocaciones científicas, Formación Profesional, vol. 35, pp.8-12.

COSCE. Confederación de Sociedades Científicas de España (2011), Informe Enciende. Enseñanza de las Ciencias en la Didáctica Escolar para edades tempranas en España, Madrid, Rubes Editorial.

GAVIDIA, V. (2008). Las actitudes en la educación científica. Didáctica de las Ciencias Experimentales y Sociales, $\mathrm{n}^{\circ} 22$, pp. 53-66.

GARCÍA, J. A., ROMERO, F. J., PERALES, F. J. y GARCÍA, R. (2010a), Visita al laboratorio de óptica: una experiencia motivadora para el alumnado de ESO y Bachillerato, Alambique, $\mathrm{n}^{\circ}$. 64, pp. 109-120.

GARCÍA, J. A., HUERTAS, R., GÓMEZROBLEDO, L., PERALES, F. J. y ROMERO, F. J. (2010b), Lo que el ojo no ve: análisis de una experiencia, Revista Eureka sobre Enseñanza y Divulgación de las Ciencias, vol. 7, pp.682-692.http://rodin.uca.es:8081/ xmlui/bitstream/handle/10498/9820/ Garca_et_al_2010.pdf?sequence $=1$

GARCÍA, J. A., GÓMEZ-ROBLEDO, L., VALERO, E., ROMERO, F. J. y PERALES, F. J. (2012), ¿Puede la divulgación científica ayudar a la labor docente universitaria?, Revista Española de Física, vol. 26, pp. 54-59.

MELLADO, V., BLANCO, J.L, BORRACHERO, A.B. y CÁRDENAS, J.A. (Eds.), Las Emociones en la Enseñanza y el Aprendizaje de las Ciencias y las Matemáticas. Badajoz: DEPROFE. 
NG,K.;LAY,Y.F.;AREEPATTAMANNIL, S.; TREAGUST, D. F. y CHANDRASEGARAN, A. L. (2012), Relationship between affect and achievement in science and mathematics in Malaysia and Singapore, Research in Science and Technological Education, vol. 30, pp. 225-237. DOI: 10.1080/02635143.2012.708655

PERALES, F.J. (2013). Actitudes hacia la Ciencia (la Tesis). Diario Granada Hoy, 14-5-2013. http://digibug.ugr. es/bitstream/10481/26026/1/ActitudesCiencia.PDF

PÉREZ, A. y DE PRO, A. (2013). Estudio demoscópico de lo que sienten y piensan los niños y adolescentes sobre la enseñanza formal de las ciencias. En V. Mellado, L.J. Blanco, A.B. Borrachero y J.A. Cárdenas (Eds.), Las Emociones en la Enseñanza y el Aprendizaje de las Ciencias y las Matemáticas (pp. 495-520), Badajoz: DEPROFE.

ROCARD, M. (Chair) (2007), Science education now: A renewed pedagogy for the future of Europe, Bruselas, Comisión Europea. http://ec.europa. e u/research/science-society/ document_library/pdf_06/reportrocard-on-science-education_en.pdf

SCHIBECI, R. A. (1986), Images of science and scientists and science education. Science Education, vol. 70, pp. 139-149. DOI: 10.1002/ sce.3730700208

SOLBES, J. (2011). ¿Por qué disminuye el alumnado de Ciencias? Alambique, $\mathrm{n}^{\circ} 67$, pp. 53-61.

SOLBES, J., MONTSERRAT, R. y FURIÓ, C. (2007). El desinterés del alumnado hacia el aprendizaje de la ciencia: implicaciones en su enseñanza. Didáctica de las Ciencias Experimentales y Sociales, ${ }^{\circ}$ 21, 91-117

VAN AALDEREN-SMEETS, S. I., WALMA VAN DER MOLEN, J. H. y ASMA, L. J. F. (2012), Primary teachers' attitude toward science: A new theoretical framework, Science Education, vol. 96, pp. 158-182. DOI: 10.1002/sce.20467

VÁZQUEZ, A. (2013). La educación científica y los factores afectivos relacionados con la ciencia y la tecnología. En V. Mellado, L.J. Blanco, A.B. Borrachero y J.A. Cárdenas (Eds.), Las Emociones en la Enseñanza y el Aprendizaje de las Ciencias y las Matemáticas (pp. 245-278), Badajoz: DEPROFE.

VÁZQUEZ, A. y MANASSERO, M. A. (2008), El declive de las actitudes hacia la ciencia de los estudiantes: un indicador inquietante para la educación científica. Revista Eureka sobre Enseñanza y Divulgación de las Ciencias, vol. 5, pp. 274-292. http://venus.uca.es/eureka/revista/ Volumen5/Numero_5_3/Vazquez_ Manassero_2008.pdf

VÁZQUEZ, A. y MANASSERO, M. A. (2009), Patrones actitudinales de la vocación científica y tecnológica en chicas y chicos de secundaria, Revista Iberoamericana de Educación, $\mathrm{n}^{\circ}$ 50, pp. 1-15. http://www.rieoei.org/ deloslectores/2950Vazquez.pdf

WhitTLE, C. (1997), Teaching science by television: The audience, education, history, and the future, ERIC (ED417079). 
ZIMEMERMAN, H.T. y BELL, B. (2012), Where Young People See Science: Everyday activities connected to
Science, International Journal of Science Education, Part B, pp. 1-29. DOI: $10.1080 / 21548455.2012 .741271$ 